Europe's Journal of Psychology, 6(3), pp. 267-287

www.ejop.org

\title{
Searching for the Sense of Humor: Stereotypes of Ourselv es and Others
}

\author{
Bernard C. Beins \\ Ithaca College
}

Shawn M. O'Toole

Ithaca College

\begin{abstract}
Researchers have made consistent claims that people do not have an awareness of their humor competence and that the vast majority of people claim to have an aboveaverage sense of humor. In this study, we examined whether people's self reports of humor competence matched an independent measurement of sense of humor. We also investigated participants' self-reported personality characteristics to see if they attributed to themselves the same characteristics that participants in earlier research attributed to hypothetical others that shared their level of humor competence. Participants completed the Multidimensional Sense of Humor Scale (MSHS) and inventories based on the Big Five model of personality. The results revealed that participants had a realistic view of their humor competence. Their self-perceived humor competence correlated reliably with their scores on the MSHS. In addition, they characterized themselves on the personality inventories in the way that previous research participants evaluated others. Participants' self-reports matched the stereotypes they had of imaginary others for extraversion and neuroticism, but not for agreeableness and openness. The findings suggest that people hold implicit theories of the link between humor and personality and apply it reliably both to themselves and to others. Results are discussed in terms of the need to study humor as multidimensional construct and in light of expectations of evolutionary theory.
\end{abstract}

Keywords - Sense of humor, stereotypes, implicit theories of personality, personality traits 
Two statements about humor appear incontrovertible. First, humor is a social lubricant. Second, having a good sense of humor is a social characteristic that people prize in themselves and others. Buss (1988) has shown that humor is highly desirable in mate selection and that people perceive it to be a highly effective strategy in mate attraction. There also seems to be a consistent relation between humor and intelligence (Howrigan \& MacDonald, 2008).

Possession of a good sense of humor $(\mathrm{SOH})$ is so important to one's self concept that, according to declarations of researchers, just about everybody believes that their sense of humor is at least average (Allport, 1961; Lefcourt \& Martin, 1986). There appear to be no reports that people are willing to state that they are truly deficient in humor, although people lower in SoH than their peers may be aware of it and provide data consistent with it (Kruger \& Dunning, 1999).

Various personality characteristics have been associated with high or low levels of humor. These associations reflect the positive view of the SoH. For example, Cann and Calhoun's (2001) participants characterized a hypothetical other regarding personality based on whether that hypothetical person had a poor, average, or good sense of humor. Systematic differences appeared as a function of putative $\mathrm{SoH}$, with a better SoH being related to more positive social traits.

People imagined as having a good sense of humor benefit from a halo effect. They are also perceived as more pleasant and interesting but less complaining or shallow (Cann \& Calhoun, 2001). Beyond this, Cann and Calhoun explored different characteristics of the NEO-FFI factors as associated with level of SoH. Again, high levels of $\mathrm{SoH}$ were related to high scores on positive factors like agreeableness, and low levels of $\mathrm{SOH}$ correlated with higher levels of neuroticism. It is important to remember that Cann and Calhoun tested participants' reactions to hypothetical others, so it is not clear that the stereotypes their participants reported are consistent with personality dimensions of actual people.

In terms of actual relations between sense of humor and personality characteristics in real people, the correlational findings are mixed. Ruch and Carrell (1998) found a fairly strong relation between trait cheerfulness and SoH. Further, Thorson and Powell (1993c) have found correlations between SoH and deference (negative) and exhibition (positive), and Kelly (2002) discovered a correlation (negative) between worry and $\mathrm{SoH}$, although the magnitude of these correlations was quite mo dest.

Other personality traits show little connection to SoH, such as achievement or aggression (Thorson \& Powell, 1993a). The lack of a relation here is not particularly 
surprising, given that sense of humor seems to consist of a relatively small number of components (Ruch \& Carrell, 1998; Thorson \& Powell, 1993a) that are probably psychologic ally unrelated to traits like achievement.

Regarding neuroticism on NEO-FFI measurements, there appears to be an inconsistent relation between neuroticism scores and level of $\mathrm{SoH}$ (e.g., Köhler. \& Ruch, 1996). Part of this inconsistency may result from an interaction between type of humor and neuroticism. Galloway and Chirico (2008) predicted that neuroticism scores and joke ratings would be related differently, depending on type of joke. Because anxiety is a component of neuroticism and because novelty is associated with anxiety, people who scored high on the neuroticism dimension would be less likely to enjoy jokes that involve novelty, that is, nonsense jokes. The researchers hypothesized, and found, that as neuroticism increases, liking of nonsense humor decreases relative to liking of incongruity-resolution humor.

The present research is based on Cann and Calhoun's (2001) investigation of evaluations of personality characteristics of hypothetic al others with varying levels of $\mathrm{SoH}$. As noted above, these researchers measured reactions to imagined people. The present research will replicate their design in the sense of relating personality characteristics to SoH. In our study, though, rather than responding about the stereotypes that people have about imagined others, participants will connect the personality traits to an actual person--themselves. As such, we will address the question of whether people with poor, typical, or good SoH show the same patterns in evaluating themselves that Cann and Calhoun's participants revealed in ev aluating others.

The answer to this question will provide insight in to the way people conceive of their own sense of humor and its relation to their personality. Given that personality researchers accept the validity of self-report measures, if our participants show the same pattern of association between level of SoH and personality in themselves as Cann and Calhoun's participants did of others, it could mean that people apply the same implicit theory of humor and personality to themselves that they do to others. One potential implication here is that participants would need to have some selfknowledge of their humor competence. Researchers have posited, however, that people do not have very good insight into their SoH (e.g., Kruger \& Dunning, 1999; Lefcourt \& Martin, 1986). Thus, if low SoH people attributed to putative others with low SoH the same personality characteristics that they themselves showed, it would imply that these people had some reliable level of insight into their own SoH. In fact, Kruger and Dunning reported a significant correlation between self reports of participants' humor competence and independently generated measures of such 
competence. On the other hand, if people have very limited insight into their humor competence, the relation between $\mathrm{SoH}$ and personality traits would be weak or nonexistent.

One goal here is to see whether our participants apply the same criteria to the mselves that Cann and Calhoun's participants applied to imagined others in their stereotypes regarding personality and humor. Further, we might see whether the stereotypes are at all veridical. That is, do the connections between participants' own SoH and their personalities resemble the stereotypical connections of Cann and Calhoun's participants?

In the present research, we will measure participants' SoH in two ways. The first is based on one question on a ten-point scale: How good is your sense of humor? This measurement is quite subjective and not tied directly to any behaviors. The second way will involve Thorson and Powell's (1993a, b) Multidimensional Sense of Humor Scale (MSHS). The MSHS is psychometrically validated and will constitute what we will refer to as an objective measurement of SoH. It is true that the MSHS involves self report, but it has acceptable psychometric properties and consists of items that are somewhat tied to descriptions of behaviors (e.g., Other people tell me I say funny things). So for convenience of description, we will refer to MSHS scores as objective rather than with more cumbersome terminology like psychometrically derived. A second, subjective single-question measurement will ask participants how funny they think they are.

The MSHS appears to have broad utility, as shown with Portuguese students (Jose, Parreira, Thorson, \& Allwardt, 2007), with Spanish students (Carbelo-Baquero, AlonsoRodriguez, Valero-Garces, \& Thorson, 2006), and with regionally diverse samples within the United States (Romero, Alsua, Hinrichs, \& Pearson, 2007). Thorson, Powell, Sarmany-Schuller, and Hampes (1997) also documented the wide range of demographic samples to which it has been administered successfully. Thus, it seems reasonable to assume that the sample in this study is appropriate for its use.

Of particular interest in the present study are both the total SoH score and the subscale scores of the MSHS that Thorson and Powell (1993) identified: humor production, coping with humor, attitudes toward the use of humor, and humor appreciation (p. 802). Given that humor is a complex construct, it seems reasonable to believe that there are multiple components to it. Research has supported the existence of the dimensions posited by Thorson and Powell. For example, Romero et al. (2007) have documented different demographic variables that correlate with the subscales of the MSHS. Further, Carbelo-Baquero et al., 2006) have used the 
subscales to distinguish humor patterns across Spanish and American samples, with the former using coping humor and the latter producing more humor. The different dimensions of the MSHS have also shown links to personality characteristics among an American sample; for instance, the humor creation and the coping subscales of the MSHS correlate reliably to hope, as measured by the Hope Scale (Cann \& Etzel, 2008).

In addition, the different dimensions of the MSHS may have some predictive power regarding issues of stress. For instance, Moran and Hughes (2006) reported that people with high humor production scores on the MSHS showed lower stress than people who tended not to produce humor. Furthermore, people scoring high on the liking of humor scale showed some higher stress scores than those who produced humor, a finding that Cann and Etzel (2008) also reported.

These dimensions can provide a more fine-grained analysis of the structure of the $\mathrm{SoH}$. Thus, possible reasons for the inconsistent relation between neuroticism scores and SoH (e.g., Deaner \& McConatha, 1993; Köhler, \& Ruch, 1996) might emerge if some, but not all, dimensions of humor relate to neuroticism. Howrigan and MacDonald (2008) and others have also shown a correlation between $\mathrm{SoH}$ and extraversion. The latter trait is embedded in the total MSHS score, but the extraversion is likely to correlate with humor production, whereas it may not relate to the use of humor for coping or to humor appreciation.

It should be noted that the MSHS may show some instability in its factors. Factors associated with relatively few items on the 24-item scale can be unreliable (Kirsch \& Kuiper, 2003). Thus, some items may tap a given underlying dimension and, depending on the sample, also show an association with different factors. In addition, as Kirsch \& Kuiper pointed out, the MSHS and other humor scales tend to focus more on the positive aspects of humor (e.g., coping) than on negative aspects (e.g., mean-spirited humor). Thus, MSHS may be useful for studying various dimensions of humor while remaining silent on others.

A second component of our study involves whether people are willing or able to report their level of SoH accurately. Claims persist that they cannot or will not (Allport, 1961; Cann \& Calhoun, 2001; Kruger \& Dunning, 1999). If people cannot report their own SoH accurately, their stereotypes of imagined others may reflect a comparison of their self image to the hypothetical other, believing themselves to be above average. As such, Cann and Calhoun's participants might have been assuming that their $\mathrm{SoH}$ is above average and, consistent with the need to hold a positive self image, might have rated positiv ely others like the $m$ (i.e., people with above-average 
SoH) and more negatively people not like them (i.e., people with below-average $\mathrm{SOH})$.

Unfortunately, the oldest report that describes everybody's belief that he or she is above average provides sketchy detail about the phenomenon. Allport's (1961) claim referred to an unpublished study in which $94 \%$ of people claimed to have SoH that was above average (p. 292-293); the methodology is unknown.

In contrast, Lefcourt and Martin (1986) provided the important elements of their research methodology. They used a five-point scale on which participants responded. The researchers reported that, in response to the item How would you rate yourself in terms of your likelihood of being amused and of laughing in a wide variety of situations? (p. 27), $94 \%$ of participants reported being at or above average, the same percentage as in Allport's work.

Additional well-documented evidence suggesting a disconnect between a person's beliefs and the reality about his or her SoH involved low SoH participants showing poor levels of agreement with the judgment of actual comedians about funniness of jokes (Kruger \& Dunning, 1999). In that research, the investigators assessed SoH based on the correlation between funniness ratings by participants and by comedians: A low correlation was equated with poor SoH in that one would expect professional comedians to have a good SoH and be able to differentiate reliably stimuli that were funny and those that were not.

Their results reveal one of the difficulties in measuring the funniness of jokes and relating it to $\mathrm{SoH}$. When Kruger and Dunning intercorrelated the ratings of the funniness of jokes by comedians, there was a clear positive relationship $(r=.72)$, but the relation was far from perfect because of the complexities involved in judging humor. In fact, the ratings of one come dian correlated negatively with the ratings of the others.

It might also have been the case that the comedians recognized the humor as coming from a particular source. The researchers chose well-known comedians (e.g., Woody Allen, Al Franken), whose work other professional comedians might recognize. For instance, the stimulus with the highest rating was "If a kid asks where rain comes from, I think a cute thing to tell him is "God is crying." And if he asks why God is crying, another cute thing to tell him is "probably because of something you did." It would not be surprising that professional comedians would recognize this humor as one of Jack Handy's "Deep Thoughts". The expectation of funniness may have been raised for those stimuli, a process that could lead to elevated ratings 
(e.g., Wimer \& Beins, 2008) both by comedians and by participants with a good SoH who might be somewhat familiar with the comedians.

Further, the differences in types of humor and people's preferences for them are always problematic and can lead to uncertainty in measurement. For instance, Kruger and Dunning did not indicate a gender breakdown among their participants. Given that more women participate in psychology studies and that women tend to show higher neuroticism scores than men (e.g., Buchanan, Johnson, \& Goldberg, 2005), the nature of the humor in that study might have had an effect on the outcome. So the correlation they reported relating assessment of one's SoH may have underestimated the participants' ability to do so.

Our methodology is similar to that of Cann and Calhoun (2001) regarding the nature of our stimuli. We assessed participants' levels of traits from the so-called Big Five, as measured by scales developed by the International Item Personality Pool (http://ipip.ori.org/ipip/). Unfortunately, for technical reasons, measurements of conscientiousness failed to record. In addition, we obtained self-reports on 36 personality characteristics that Cann and Calhoun measured (e.g., friendly, interesting, complaining, passive). Those researchers identified the characteristics on two orthogonal dimensions: desirability (high or low) and control (high or low).

The difference between their study and ours is that our analysis will investigate whether participants with a good sense of humor will show the same characteristics that Cann and Calhoun's participants envisioned in a person with a good sense of humor. That is, do people attribute the same stereotypic al characteristics associated with a good sense of humor in others to themselves? Similarly, do those with a poor sense of humor do so?

Kruger and Dunning (1999) reported that their participants' self-ratings of humor compared to their peer group correlated significantly with those researchers' objective measurement of $\mathrm{SoH}$. We are looking at whether participants are aw are of their level of $\mathrm{SoH}$ as measured on a Likert-type scale without reference to a peer group. Previous self-measurements of $\mathrm{SoH}$ may have relied on less specific measurements given the report that $94 \%$ of participants believed that they were above average (Allport, 1961). In addition, it is not clear how participants differ in their answers if they compare themselves to a peer group as opposed to simply assessing the mselves via a number on a more abstract Likert-type scale. 


\section{Method}

Participants

We tested 109 volunteers from psychology courses. They received extra credit for their participation. There were 75 women and 33 men. The sample comprised 97 white participants, 6 black participants, 4 Asian Americans, and 2 who did not indicate race or ethnicity.

Materials

We used measures of the Big Five personality traits from the Collaboratory provided by the Oregon Research Institute (http://ipip.ori.org/ipip/). They included 10-item scales for neuroticis $m$ with an acceptable level of reliability $(a=.86$ ), extraversion ( $a$ $=.86)$, and openness ( $a=.82)$; we used the 20-item scale agreeableness $(a=.85)$ because its reliability was comparable to that of the other scales.

In addition, our participants completed the Multidimensional Sense of Humor Scale (MSHS; Thorson and Powell, 1993a), a 24-item self-report inventory with statements like My clever sayings amuse others and Humor is a lo usy coping mechanism (reverse scored). Responses to items on this inventory are on a scale of 1 (Very inaccurate) to 5 (Very accurate). For data analysis to replic ate Cann and Calhoun's approach, we created three levels of SoH: low, medium, and high. Our cutoffs for inclusion in the three groups involved a breakdown into three groups with approximately equal numbers of participants based on scores on the MSHS.

Next, participants rated themselves on the set of 36 adjectives that Cann and Calhoun (2001, p. 122) used. The adjectives reflected traits that represented high or low desirability and high or low control. High desirability-high control adjectives included words like friendly and pleasant. High desirability-low control words included interesting and imaginative. The low desirability-high control words inv olved words such as complaining and cold; low-desirability-low control included words such as passive and restless. Participants self-rated these on the same scale of 1 (Not at all like me) to 9 (Completely like me) that Cann and Calhoun used.

Procedure

Participants reported to the laboratory and completed the study in groups either on a computer or on paper. They sat in nonadjacent seats to minimize the likelihood 
that responses would be influenced by others who might see how they rated the mselves on the various characteristics.

After completing informed consent forms, they rated themselves on measures of neuroticism, extraversion, agreeableness, and openness. Subsequent to those ratings, participants completed the Multidimensional Sense of Humor Scale, then rated the mselves on the 36 adjectives that differed in control and desirability.

\section{Results}

\section{Subjective and Objective Measures of Humor}

When our participants indicated on a 10-point scale how funny they thought they were and how much of a SoH they possessed, the results showed, predictably, that they tended not to rate the mselves as being at the very low end of the scale. But the 10-point scale gave them the psychological space to indicate that they believed the mselves to be low relative to others. In fact, for both funniness and SoH, the data are reasonably well distributed. Although it is not surprising that people did not rate themselves as being entirely humorless, few people rated themselves at the highest levels for either characteristic. The patterns appear in Figures 1 and 2.

These data belie the claim that people are not aware of their humorous aspects. In fact, both Lefcourt and Martin (1986) and Kruger and Dunning (1999) reported significant correlations between inventory items or other external measures of sense of humor and self-ratings. The present data resulting from a 10-point scale shows that people are clearly not oblivious to their relative standing regarding humor competence. In fact, if one were to ignore self ratings of three and lower, an area on the scale that is sparsely populated, one has a typical seven-point Likert-type scale with ratings along the entire scale.

Particularly for $\mathrm{SoH}$, people are reluctant to place themselves at the very bottom of the scale. But they are willing to admit that they are not at the top of the scale.

Using our objective measure of SoH, the MSHS, we grouped participants as being low, medium, or high in SoH to match the categories that Cann and Calhoun used for the assessment of hypothetical others. When we analyzed our participants' self reports about sense of humor, there was remarkable agreement between the participants' self perceptions and the objective measurements for their responses to both self assessments, "How funny do you think you are?" and "How would you rate 
your sense of humor?"

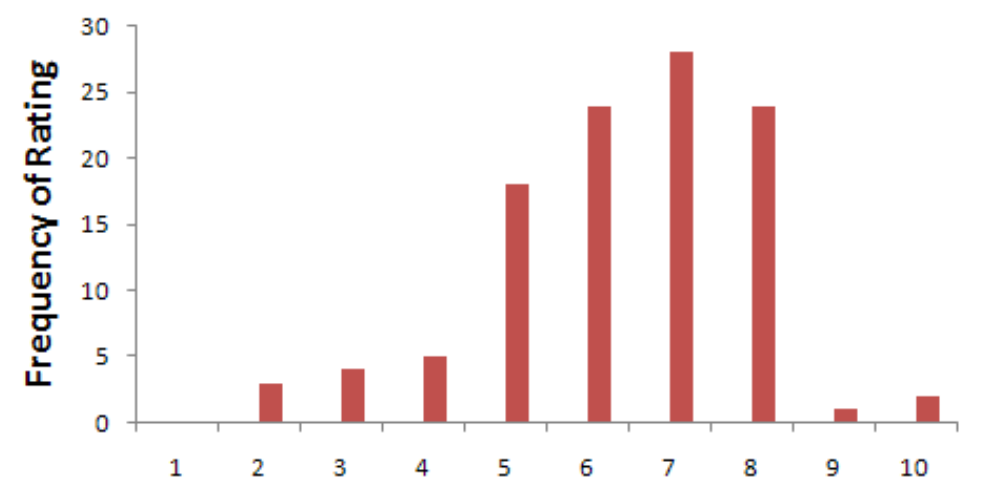

Self Rating of Funniness

Figure 1. Frequency of occurrence of self rated funniness on a scale of 1 (Not very funny) to 10 Very funny)

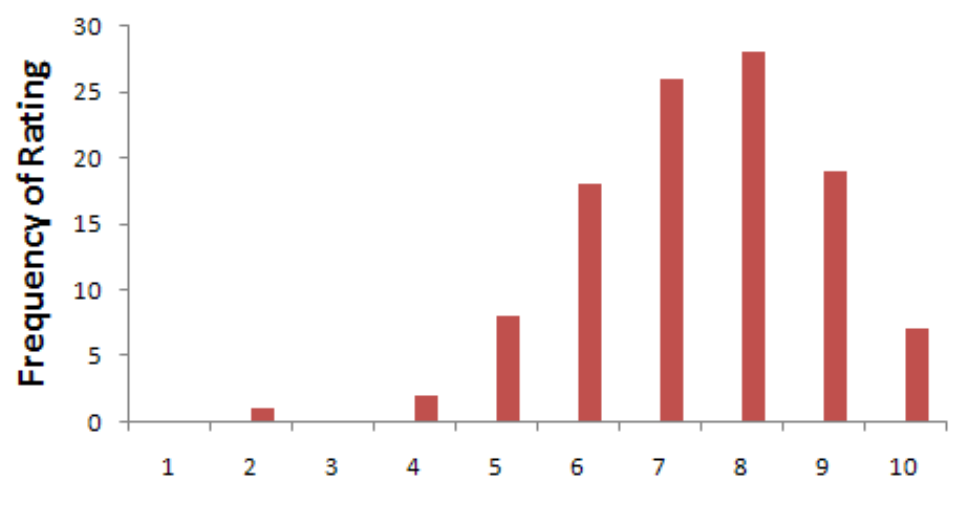

Self Rating of Sense of Humor

Figure 2. Frequency of occurrence of self rated sense of humor on a scale of 1 (Very poor sense of humor) to 10 (Verygood sense of humor)

Participants who reported not being very funny scored reliably lower on the MSHS, those seeing the mselves as being moderately funny scored in the middle, and those whose self perceptions were as being very funny scored highest, $F(2,103)=23.67, p<$ .01 , partial $\eta^{2}=.32$. Participants who reported not having much of a SoH were likely to score lowest on the MSHS, with those reporting a moderate SoH reliably higher on the MSHS, and those claiming best SoH scoring highest on the MSHS, $F(2,103)=7.62$, $p<.01$, partial $\eta^{2}=.13$. The pattern of results appears in Figure 3. For each question, the self-reported humor in the low, medium, and high groups differs significantly from the other two groups. 


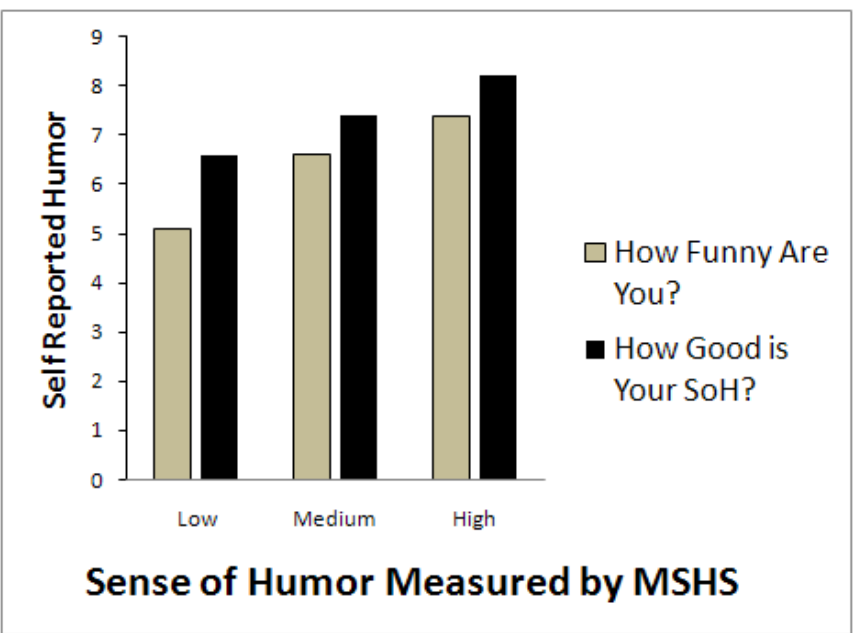

Figure 3. Self-reported humor levels as a function of level of humor competence as measured by the MSHS

We factor analyzed the 24 items from the MSHS into its components. The factor analysis with Varimax rotation revealed five components with eigenvalues greater than 1.00. Although our sample size was on the lower bound for analyzing a 24-item instrument, our factors were similar enough to those of Thorson and Powell (1993a) that we are comfortable using the factors that emerged from the analysis. Together, they accounted for $67.04 \%$ of the variance.

The factors involved (a) humor production (items 1 to 10), (b) positive feeling about coping humor (items 11 to 14 and item 16), (c) utility of humor (items 15 and 17), (d) humor appreciation (items 19 to 23), and (e) comfort with humor (items 18 and 24). All coefficients but one were above .5; the single exception was .45. Table 1 gives examples of some of the MSHS items associated with the five factors.

There is good consistency regarding participants' views of their own humor competence and its objective measurement on the MSHS. Reinforcing these results is the significant correlation between self reports of being funny and the MSHS score, $r(107)=.71, p<.01$. The correlation between the self report and the humor production factor on the MSHS was even higher, $r(107)=.77, p<.01$.

With respect to the self report of having a good sense of humor, participants' self ratings correlated signific antly with MSHS scores, $r(107)=.44, p<.01$. The phrase sense of humor appears to mean something different than being funny, given this lower correlation with MSHS score and with the even lower correlation between selfreported SoH and MSHS score on the production factor, $r(107)=.42, p<.01$. 


\begin{tabular}{ll}
\hline Factor & Example \\
\hline $\begin{array}{l}\text { Humor Production } \\
\text { Positive Feelings about Coping } \\
\text { Humor }\end{array}$ & $\begin{array}{l}\text { I'm regarded as something of a wit by my } \\
\text { friends }\end{array}$ \\
$\begin{array}{ll}\text { Utility of Humor } \\
\text { Humor Appreciation }\end{array}$ & Humor is a lousy coping mechanism \\
Comfort with Humor & I appreciate those who generate humor \\
& I'm uncomfortable when everyone is \\
\hline
\end{tabular}

Table 1. Examples of items from the MSHS (Thorson \& Powell, 1993a) associated with the five factors resulting from a factor analysis of participant responses

In general, there is a stronger relation between MSHS score with its components and belief in one's funniness than belief in one's SoH. So either the MSHS is a better measure of funniness as people characterize it, or SoH is a more global construct that goes beyond the factors of the MSHS. Still, the MSHS does more than an adequate job of dealing with both perceived funniness and $\mathrm{SOH}$.

Personality Characteristics and Humor

When we analyzed our data using the same nominal groupings that Cann and Calhoun did (low, average, and high in $\mathrm{SoH}$ ) when their participants envisioned a hypothetical other, there were some similarities in the patterns of results. For some personality characteristics, our participants rated their own personality the same way Cann and Calhoun's participants rated a hypothetical other. Our participants attributed extraversion to themselves just as Cann and Calhoun's participants attributed extraversion to the hypothetical other, with low-SoH participants showing significantly lower levels of extraversion than medium or high, $F(2,103)=6.03, p<.01$, partial $n^{2}=.10$

With respect to neuroticism, a marginally significant effect showed that our participants with low and medium SoH were about equal, with high SoH participants having lower levels of neuroticism, $F(2,103)=2.40, p=.10$, partial $\eta^{2}=.04$. This marginal effect replicates that of Cann and Calhoun. 
Our pattern of results regarding openness shows some similarity with the previous research, with the highest levels of openness associated with high-SoH participants, $F(2,103)=3.05, p=.05$, partial $\eta^{2}=.06$, although the levels for low and medium participants is reversed here compared to Cann and Calhoun. Finally regarding this set of characteristics, we found no differences across humor levels regarding agreeableness, $F<1$. Cann and Calhoun found increasing levels of agreeableness attributed to others as their putative level of SoH increased. Summary data from our study appear in Table 2.

\begin{tabular}{lllll}
\hline & Extraversion & Neuroticism & Openness & Agreeableness \\
\hline Low & $3.19(.12)$ & $2.41(0.11)$ & $3.46(0.09)$ & $3.67(0.09)$ \\
Medium & $3.81(.13)$ & $2.31(0.12)$ & $3.26(0.10)$ & $3.73(0.10)$ \\
High & $3.60(.12)$ & $2.64(0.11)$ & $3.58(0.09)$ & $3.56(0.08)$ \\
\hline
\end{tabular}

Table 2. Mean self ratings of personality characteristics according to level of sense of humor as measured by the MSHS

These results are intriguing in that the attribution of some personality characteristics is similar regardless of whether one is evaluating a hypothetical other with, for instance, an above-average SoH or a high-SoH person is evaluating himself or herself. Such attributions occurred both for positive traits (i.e., extraversion and openness) and a negative trait (i.e., neuroticism).

In addition to analyzing overall humor competence, we investigated the relation between subcomponents of humor, as identified in the factor analysis of the MSHS data, and the elements of the Big Five personality theory. The patterns of correlation appear in Table 3. Interestingly, overall humor competence did not correlate with neuroticism for either funniness or $\mathrm{SoH}, r=-.06$ and .04 , both $\mathrm{ps}>.56$. Nor was there a relation between funniness or SoH and openness, $r=.08$ and .03 , both ps $>.41$. (For all analyses, $d f=107$.)

Self-reported funniness and extraversion were significantly correlated, $r=.35, p<.01$, whereas $\mathrm{SoH}$ was marginally related, $r=.18, p=.06$. Agreeableness and funniness were not significantly correlated, $r=.11$, but agreeableness and SoH were, $r=.20, p$ $=.04$. These latter two patterns suggest that the psychological processes associated with funniness and SoH may overlap but that they also have discrete components. 
A breakdown of MSHS factors and their associations revealed that certain personality characteristics are correlated with different traits. For instance, humor production correlated signific antly with extraversion, but not with any other traits.

\begin{tabular}{lcccc}
\hline & Extraversion & Neuroticism & Openness & Agreeableness \\
\hline Humor Production & $.37^{* *}$ & .03 & .10 & .06 \\
Coping with Humor & .00 & -.03 & $.27^{* *}$ & $.27^{*}$ \\
Utility of Humor & -.02 & -.12 & -.11 & $.23^{*}$ \\
Humor Appreciation & .03 & -.08 & $.26^{* *}$ & $.29^{*}$ \\
Comfort with Humor & -.11 & $-.32^{* *}$ & -.06 & $-.21^{*}$ \\
Total MSHS Score & $.24^{*}$ & -.06 & $.24^{*}$ & .08 \\
\hline
\end{tabular}

Note: Significant correlations appear in bold type. For all correlations, $d f=107$. ${ }^{*} p<.05 . \quad * * p<.01$.

Table 3. Correlations between personality traits and components of sense of humor

These correlations provide an interesting look at the various components of humor and the traits associated with them. Participants with high extraversion scores produce humor, but their extraversion does not predict anything about the other components of humor. Similarly, participants scoring high on neuroticism show discomfort with humor, agreeing that statements like I'm uncomfortable when everyone is cracking jokes describe them accurately.

Another pattern of interest concerns openness. There is a direct relation between one's level of openness and the two components of humor, use of coping humor and humor appreciation. Openness does not correlate with negative responses to humor, discomfort or how useful humor is for coping. As such, openness relates to positive reactions to humor but not to negative responses. This provides an interesting contrast to the decreasing comfort with humor as neuroticism increases without a concomitant relation to responses to positive use of coping humor.

In addition, agreeableness is not associated with humor production, but it is with acceptance of and appreciation of humor. This is not surprising given that agreeableness in our measurements was associated with a positive outlook, which could reasonably include appreciating the value of humor and its ameliorative social effects. 
Finally, the ratings of the adjectives that varied in control and in desirability did not vary systematic ally with humor level, as in Cann and Calhoun's study. We presented all adjectives in each category together, which we think simply led to higher ratings for high desirability adjectives and lower ratings for low desirability adjectives without differentiating across humor levels. Thus, we will not discuss this analysis further.

\section{Discussion}

The two most interesting and important findings in this research are that (a) people do know their level of humor competence and will report it with reasonable accuracy and (b) people attribute some traits to themselves that they attribute to a hypothetical other who possesses the same level of humor competence as they do. As noted above, a recurrent the me in discussing humor competence is that people believe that they show high levels of sense of humor and that they report their levels inaccurately (Allport, 1961; Kruger \& Dunning, 1999; Lefcourt \& Martin, 1986). This the me persists in spite of reports correlations between self-perceived SoH and some objective measures of SoH (Kruger \& Dunning; 1999; Lefcourt \& Martin, 1986).

One of the difficulties in measuring personality and humor is the subjectivity that is necessarily involved. There is no objective metric for identifying constructs of funniness of jokes (Wimer \& Beins, 2008), so using responses to jokes as a measurement of a person's sense of humor is reasonable, but not perfect. Similarly, measuring a person's self perception of humor has its limitations because such measurement implies that people are able and willing to monitor the effect that their humor production has on those around them. Our data reflect the importance of the particular measurements used and the need to attend to the multidimensional nature of humor.

In this study, we extended the scale of possible responses from the typical five as used by Lefcourt \& Martin to a ten-point scale. Knowing that people are unwilling to characterize the mselves as completely devoid of humor competence, we reasoned that by extending the scale, we would allow people to avoid assigning extremely low values to their level of humor but, at the same time, they might still be able to position themselves with relative accuracy. Our results justified that approach. Using a ten-point scale appears in a psychological sense to be the same as using a sevenpoint scale on which there are three fictitious (i.e., unused) points. Participants tended not to self rate with very low numbers when reporting their $\mathrm{SoH}$, although they were willing to admit being low on the funniness scale. It appears that people differentiate between having a good $\mathrm{SoH}$, which is highly desirable, and being funny, which is also desirable but not absolutely critical. 
When we examined participants' scores on the MSHS in relation to their self-reported $\mathrm{SoH}$ and level of funniness, we found impressive correlations. The correlation between the humor production factor of the MSHS and the self rating of funniness was .765 , which would account for over $58 \%$ of the variance in that relation. This figure is very high for measurements of such a complex psychological construct.

The aforementioned research implies that some cognitive characteristics are associated with recognition of one's humor competence. Kuiper, McKenzie, and Belanger (1995) showed that humorous people were able to change their perspectives when contexts changed; they called for recognition of the multidimensional nature of humor as it related to individual differences. Other researchers have taken their cues from Kuiper et al. in the realm of personality characteristics (e.g., Craik, Lampert, \& Nelson, 1996; Köhler, \& Ruch, 1996).

The research addressing the association of humor and personality characteristics is very complex and sometimes inconsistent. For example, neuroticism sometimes correlates with SoH (e.g., Deaner \& McConatha, 1993), but sometimes it does not (e.g., Köhler, \& Ruch, 1996). In the present research, neuroticism did not correlate with overall $\mathrm{SoH}$ as measured by the MSHS, but higher scores on neuroticism are associated with greater levels of discomfort with expressions of humor. As Köhler \& Ruch (1996) pointed out, measures of neuroticism focus on qualities like shyness and mood changes; further, Gallow ay and Chirico (2008) pointed out that people high in neuroticism dislike the anxiety of some humor (e.g., nonsense humor), so it is no surprise that humor production is unrelated to neuroticism score in our data. Thus, the nature of the measurement appears to be crucial in establishing any connection among these variables, as suggested by Kuiper at al. (1995). This suggestion is further supported by our finding that the relations between total MSHS scores and personality scores are not as strong as the relations between subcomponents of the MSHS and personality scores. Looking back at Lefcourt and Martin's (1986) report that $94 \%$ of participants were at or above average in humor, one can ask whether the question to which they referred may relate to humor appreciation or coping more than to other subcomponents of humor.

In fact, Dewitte and Verguts (2001) showed that what constitutes a "good joker" involves two components, frequent joking and attention to the effects of that joking. Self-monitoring seems to be a critical element in becoming a good joker because a degree of sensitivity to listener reactions helps fine tune one's humor skills. As such, the importance of self monitoring may explain why openness and agreeableness are reliably associated with $\mathrm{SoH}$ : Both of these characteristics relate to paying attention to context. 
The importance of separating the components of humor is clear in our data regarding openness and agreeableness. MSHS overall scores correlate significantly with agreeableness scores but not with openness as shown in Table 3. A breakdown into the components of humor, on the other hand, reveals an interesting relation between neuroticism scores and the comfort with humor subcomponent of the MSHS. The lack of an overall correlation between MSHS score and neuroticism score fails to reveal that those high in neuroticism feel discomfort around the expression of humor, a finding also reported by Köhler, \& Ruch (1996).

Extraversion is reliably associated with humor production, although it is not clear that extraversion is association with being a good joker. If an extravert is low in social sensitivity, the person may tell jokes frequently, but not well because the person has not used cues from the audience to refine his or her ability, as Dewitte and Verguts noted.

Thus, even though there may be no everyday, objective metric for measuring humor or one's sense of humor, those high in self-monitoring may pick up reliable cues from those around them. In fact, Turner (1980) found that research participants rated high self monitors as being wittier; participants similarly rated cartoon captions produced by that group as funnier than captions produced by low self monitors. It is possible that those lower in sensitivity to pick up on social cues may constitute the group that sees itself, erroneously, as above average in funniness; they observe themselves telling jokes, but they do not register the lack of success in their performance. This research also provided reason that people have at least some ability to recognize their humor competence.

Another aspect of our research addressed the degree to which participants' self perceptions tended to match their perceptions of people who were like them in terms of level of humor competence. Cann and Calhoun reported on stereotypes that people have of others with below average, typical, and above average senses of humor; we investigated whether participants attributed to themselves the same characteristics that Cann and Calhoun's participants attributed to an imaginary other. Our results revealed that there were similarities between self ratings of participants with a given level of humor competence and Cann and Calhoun's reports of ratings of hypothetical others. This comparability suggests that the attributions accorded to others who may have, for example, a very good sense of humor are actually shared by people whose objectively determined sense of humor is good. 
Perceptions of one's humor level may not be perfect, nor are objective measurements, but there is an element of veridicality to both. Cann and Calhoun reported that imaginary others with a good sense of humor were viewed as being more intelligent than those with a poor sense of humor. As Howrigan and MacDonald (2008) have shown, there is a reliable correlation between intelligence and humor and between extraversion and humor (the latter being a consistent finding). So there is little surprise to see some correlation between intelligence and extraversion. Nor is it a surprise that people would correctly perceive this link.

One might suspect that spotting positive traits like intelligence (and their correlates) might be matched by the ability to spot undesirable traits. Our results match those of Cann and Calhoun regarding neuroticism. Our participants attributed to themselves the same level of neuroticism that Cann and Calhoun's participants attributed to imaginary others when the level of humor competence of our participants matched that of Cann and Calhoun's imaginary other.

In contrast, our participants did not show different levels of openness or agreeableness as a function of level of humor competence. This lack of an effect probably would not surprise either Howrigan and MacDonald (2008) or Buss (1988), who advanced their arguments in the context of evolutionary psychology, specifically mate selection. Higher levels of humor are associated with "a positive growth-oriented fashion with a variety of life circumstances and situations" (Kuiper et al., 1995, p. 371). One can imagine that neither openness nor agreeableness per se would be evolutionarily related to mate selection in the same way as intelligence, which may be reliably associated with perceptions of humor competence through the related variable of extraversion. It would be useful to explore the possible connection between perceptions of intelligence, humor, and extraversion.

In conclusion, our data support the contention that people are good, but not perfect, in assessing their level of humor competence. Further, when they assess the humor competence of others (see Cann and Calhoun, 2001), they may be using their own self perceptions as a basis in evaluating others. Thus, some of the humor stereotypes of others that Cann and Calhoun reported may have their root in veridical self perceptions. The importance of humor in social situations and the ability to recognize traits that co-occur with high levels of humor competence are not unexpected when viewed within the context of evolutionary psychology. Finally, in assessing personality characteristics associated with humor competence, it is important to treat humor as the multidimensional construct that it is. 


\section{References}

Allport, G. H. (1961). Pattern and growth in personality. New York: Holt, Rinehart, and Winston.

Buchanan, T., Johnson, J. A., \& Goldberg, L. R. (2005). Implementing a five-factor personality inventory for use on the internet. European Journal of Psychological Assessment, 21, 115-127. doi:10.1027/1015-5759.21.2.115

Buss, D. M. (1988). The evolution of human intrasexual competition: Tactics of mate attraction. Journal of Personality and Social Psychology, 54, 616-628. doi:10.1037/00223514.54.4.616

Cann, A., \& Etzel, K. C. (2008). Remembering and anticipating stressors: Positive personality mediates the relationship with sense of humor. Humor: International Journal of Humor Research, 21, 157-178. doi:10.1515/HUMOR.2008.008

Cann, A., \& Calhoun, L. G. (2001). Perceived personality associations with differences in sense of humor: Stereotypes of hypothetical others with high or low senses of humor. Humor: International Journal of Humor Research, 14, 117-130. doi:10.1515/humr.14.2.117

Carbelo-Baquero, B., Alonso-Rodriguez, M. C., Valero-Garces, C., \& Thorson, J. A. (2006). A study of sense of humor in spanish and american samples. North American Journal of Psychology, 8(3), 447-454.

Deaner, S. L., \& McConatha, J. T. (1993). The relationship of humor to depression and personality. Psychological Reports, 72, 755-763.

Galloway, G., \& Chirico, D. (2008). Personality and humor appreciation: Evidence of an association between trait neuroticism and preferences for structural features of humor. Humor: International Journal of Humor Research, 21, 129-142. doi:10.1515/HUMOR.2008.006

Greengross, G., \& Miller, G. F. (2009). The big five personality traits of professional comedians compared to amateur comedians, comedy writers, and college students. Personality and Individual Differences, 47, 79-83. doi:10.1016/j.paid.2009.01.045

Howrigan, D. P., \& MacDonald, K. B. (2008). Humor as a mental fitness indicator. Evolutionary Psychology, 6, 625-666. 
José, H., Parreira, P., Thorson, J. A., \& Allwardt, D. (2007). Factor-analytic study of the multidimensional sense of humor scale with a portuguese sample. North American Journal of Psychology, 9, 595-610.

Kelly, W. E. (2002). An investigation of worry and sense of humor. The Journal of Psychology, 136, 657-666.

Kirsh, G. A., \& Kuiper, N. A. (2003). Positive and negative aspects of sense of humor: Associations with the constructs of individualism and relatedness. Humor: International Journal of Humor Research, 16, 33-62. doi:10.1515/humr.2003.004

Köhler, G., \& Ruch, W. (1996). Sources of variance in current sense of humor inventories: How much substance, how much method variance? Humor: International Journal of Humor Research, 9, 363-397.

Kruger, J., \& Dunning, D. (1999). Unskilled and unaware of it: How difficulties in recognizing one's own incompetence lead to inflated self-assessments. Journal of Personality and Social Psychology, 77, 1121-1134. doi:10.1037/0022-3514.77.6.1121

Lefcourt, H. M., \& Martin, R. A. (1986). Humor and life stress: Antidote to adversity. New York: Springer/Verlag.

Moran, C., \& Hughes, L. P. (2006). Coping with Stress: Social Work Students and Humour. Social Work Education, 25, 501-517. Doi: 10.1080/02615470600738890

Romero, E. J., Alsua, C. J., Hinrichs, K. T., \& Pearson, T. R. (2007). Regional humor differences in the United States: Implications for management. Humor: International Journal of Humor Research, 20, 189-201. doi:10.1515/HUMOR.2007.009

Thorson, J. A., \& Powell, F. C. (1993a). Development and validation of a multidimensional sense of humor scale. Journal of Clinical Psychology, 49, 13-23. doi:10.1002/10974679(199301)49: 1<13::AID-JCLP2270490103>3.0.CO;2-S

Thorson, J. A., \& Powell, F. C. (1993b). Relationships of death anxiety and sense of humor. Psychological Reports, 72, 1364-1366.

Thorson, J. A., \& Powell, F. C. (1993C). Sense of humor and dimensions of personality. Journal of Clinical Psychology, 49, 799-809. doi:10.1002/1097-4679(199311)49:6<799::AIDJCLP2270490607>3.0.CO;2-P

Thorson, J. A., Powell, F. C., Sarmany-Schuller, I., \& Hampes, W. P. (1997). Psychological 
health and sense of humor. Journal of Clinical Psychology, 53, 605-619.

Turner, R. G. (1980). Self-monitoring and humor production. Journal of Personality, 48, 163-172. doi: doi:10.1111/j.1467-6494.1980.tb00825.x

Wimer, D. J., \& Beins, B. C. (2008). Expectations and perceived humor. Humor: International Journal of Humor Research, 21, 347-363. doi:10.1515/HUMOR.2008.016

About the authors:

Bernard C. Beins

Bernard C. Beins is professor of Psychology. He chairs the Psychology Department at Ithaca College in Ithaca, New York. His interests involve the relation between personality and sense of humor, the role of expectations in humor perception, the scholarship of teaching and learning, and the history of psychology.

Address for correspondence: Prof. Bernard Beins, School of Humanities and Sciences, 119 N Williams Hall Ithaca, NY 14850

E-mail: beins@ithaca.edu

Shawn O'Toole

Shawn M. O'Toole is a 2007 graduate of Ithaca College, where he earned his B.A. in Psychology. Currently, he is pursuing his Master's Degree in Adolescent Education at St. John's University. Shawn ultimately plans to teach both Mathematics and Special Education. Correspondence with the author: shawnot@gmail.com. 